\title{
Françoise Rubellin (sous la direction de), Théâtre de la Foire. Anthologie de pièces inédites (1712-1736)
}

\section{Franco Piva}

\section{Q OpenEdition}

\section{Edizione digitale}

URL: https://journals.openedition.org/studifrancesi/26262

DOI: $10.4000 /$ studifrancesi.26262

ISSN: 2421-5856

\section{Editore}

Rosenberg \& Sellier

\section{Edizione cartacea}

Data di pubblicazione: 1 avril 2007

Paginazione: 175

ISSN: 0039-2944

\section{Notizia bibliografica digitale}

Franco Piva, «Françoise Rubellin (sous la direction de), Théâtre de la Foire. Anthologie de pièces inédites (1712-1736)», Studi Francesi [Online], 151 (LI | I) | 2007, online dal 30 novembre 2015, consultato il 23 novembre 2021. URL: http://journals.openedition.org/studifrancesi/26262 ; DOI: https://doi.org/ 10.4000/studifrancesi.26262

Questo documento è stato generato automaticamente il 23 novembre 2021.

\section{(c) (1)}

Studi Francesi è distribuita con Licenza Creative Commons Attribuzione - Non commerciale - Non opere derivate 4.0 Internazionale. 


\title{
Françoise Rubellin (sous la direction de), Théâtre de la Foire. Anthologie de pièces inédites (1712-1736)
}

\author{
Franco Piva
}

\section{NOTIZIA}

FRANÇOISE RUBELLIN (sous la direction de), Théâtre de la Foire. Anthologie de pièces inédites (1712-1736), Montpellier, Editions Espace 34, 2005, pp. 417.

1 Il Teatro della Foire, dopo gli studi pionieristici di Marcello Spazioni, e quelli, più recenti, di Dominique Lurcel, Renzo Guardenti, Derreck Connon, George Evans, David Trott e Isabelle Martin, per non citare che i più noti ed importanti, è, come fenomeno sociale e letterario, ormai abbastanza ben conosciuto. Meno sicure sono, a tutt'oggi, le nostre conoscenze per quanto riguarda il suo repertorio. Di fondamentale importanza, il Théâtre de la Foire pubblicato da Lesage e d'Orneval negli anni Trenta del Settecento, e su cui si sono basati per molto tempo coloro che al Théâtre della Foire si sono accostati, è infatti troppo centrato sull'attività di questi due autori. Le antologie più recenti, come quelle pubblicate da Marcello Spaziani, Dominique Lurcel, Derreck Connon e George Evans, hanno certamente ampliato questo repertorio, e hanno fatto vedere come questo teatro abbia coinvolto più autori, ed abbia comportato forme fino ad allora insospettate. La presente antologia, che il Centre d'Etude des Théâtres de la Foire dell'Università di Nantes ha approntato sotto la sapiente direzione di Françoise Rubellin, apporta alla nostra conoscenza del Théâtre de la Foire un nuovo importante tassello: non solo perché allarga il repertorio finora noto con 11 pièces inedite, ma anche perché conferma come il ventaglio degli autori che scrissero per il Théâtre de la Foire sia assai più ampio di quanto si fosse potuto supporre, anche dopo gli studi e le raccolte precedentemente citati: accanto ai nomi, già noti, di Lesage, d'Orneval, Biancolelli e Fuzelier, troviamo infatti quelli, certamente meno noti se non del tutto sconosciuti, di Desgranges, Piron, Carolet, Pannard, Pontau e Parmentier. Le 
informazioni desunte dall'attenta analisi cui le diverse pièces sono state sottoposte dai giovani collaboratori di Françoise Rubellin, hanno inoltre permesso di precisare meglio i rapporti che il Teatro della Foire intrattenne non solo col Théâtre Français e con l'Opéra, ma anche con l'Ancien Théâtre Italien, di cui il Théâtre de la Foire riprese, come è noto, almeno in parte tradizione e repertorio, in forme e modi tuttavia che il presente volume precisa in maniera talvolta inattesa. Altrettanto interessanti sono le informazioni che queste pièces, e lo studio attento dei manoscritti che le contengono, ci offrono circa le condizioni nelle quali esse vennero rappresentate, i décors, a volte sorprendenti, che furono utilizzati, i rapporti, spesso conflittuali, che caratterizzarono la vita delle diverse troupes, i generi via via praticati o inventati, anche sulla scorta e a seguito dei burrascosi rapporti che il Teatro della Foire intrattenne, come è noto, coi cosiddetti teatri ufficiali, che alla Foire fecero in quegli anni una guerra tanto spietata quanto inutile, visto che la fantasia dei Forains riuscì ad avere sempre la meglio. Insomma un insieme di pièces che, oltre ad allargare utilmente il repertorio finora conosciuto, offre sul o forse sarebbe meglio dire sui Théâtres de la Foire, sulla loro vita tribolata ed esaltante, sui loro attori e sui loro collaboratori tutta una serie di informazioni utili per una migliore conoscenza di un genere, quello del Teatro della Foire, che non finisce mai di stupire per la sua varietà e per la sua straordinaria vitalità. 